\author{
Dirk Beckedorf \\ Franz Müller
}

\section{Einführung}

Die Hörtherapie nach A. Tomatis (auch Audio-Psycho-Phonologie, A.P.P., genannt) ist eine auditive Behandlungsmethode. Sie erfasst und optimiert vielfältige Prozesse des Hörsinns und dessen Auswirkungen in Körper und Psyche. Begründet wurde sie von dem französischen Hals-Nasen-Ohrenarzt Dr. Alfred Tomatis in der zweiten Hälfte des 20. Jahrhunderts und wurde dann auf verschiedenen Ebenen weiterentwickelt.

\section{Übersicht über die Therapie}

Grundlagen der Behandlung sind ein spezieller Hörtest (Hörprofil) und eine ausführliche Anamnese. Die Patienten hören anschliessend mit Spezialkopfhörern ausgewählte Werke von Mozart sowie gregorianische Gesänge. Die Musik wird durch Klangwandler individuell in ihren Frequenzspektren moduliert («gefiltert»).

Die Hörtherapie folgt systemischen Aspekten im Setting wie auch in den Therapieinhalten. Kinder hören nach der Eingangsdiagnostik in einem Kinderraum im Regelfall $2 \mathrm{~h}$ pro Tag die individuell gefilterte $\mathrm{Mu}$ sik und werden dabei von den Hörtherapieassistenten begleitet. Sie dürfen in dieser Zeit ihren Impulsen folgen, feinmotorische Tätigkeiten wie Basteln oder Malen ausüben oder auch frei spielen. Ein Elternteil begleitet das Kind, indem sie oder er räumlich getrennt von dem Kind in einem Erwachsenenraum auch gefilterte Musik hört. Der Hörprozess der Kinder wird also doppelt gerahmt -

\title{
Die Hörtherapie nach Dr. Alfred Tomatis
}

durch die Hörtherapeuten und durch die Eltern. Innerhalb dieser doppelten Rahmung stimuliert die gefilterte Musik Eigenregulation, Kommunikation und Selbstfindung.

Kinder sowie Erwachsene hören im Regelfall in mehreren intensiven Hörabschnitten. Am Anfang stehen an 15 aufeinanderfolgenden Tagen täglich 2 h Hörtherapie. Nach mehrwöchigen Pausen folgen üblicherweise weitere Hörabschnitte von 8-10 Tagen mit täglich 2 h Hören. Die Frequenzveränderung der Musik folgt einem systematischen Aufbau, der individuell nach Hörprofil und Therapieauftrag angepasst wird. Dabei wirkt die Musik auf die beiden im Innenohr auf engstem Raum vereinten Sinne ein: das Gleichgewichtsorgan und die Hörschnecke (Cochlea).

\section{Therapieinhalte}

Hören ist ein intensiv körperlich wahrgenommenes Geschehen: Klänge können das Herz öffnen, einen Schauer über den Rücken jagen, kribbelig machen, Bewegungsimpulse wecken oder dämpfen. Sie können auch Körper und Geist beruhigen sowie Sammlung und Konzentration bewirken. Vielfältig sind die Auswirkungen des Hörens auf Körper und Psyche.

\section{Anregung von Tiefenentspannung, \\ Aufmerksamkeit und Wachheit}

Der Hörsinn ist eingebettet in grundlegende Funktionen des autonomen Nervensystems mit seinen beiden Ästen - dem bindungsstärkenden Parasympathikus und dem anspannenden und erregenden Sympathikus.
In dieses Wechselspiel greift das zentrale Wirkprinzip der Hörtherapie, die «Klangwippe», modulierend ein. Das Wirkprinzip der Klangwippe besteht in einer wechselnden Betonung tiefer und hoher Frequenzen, die durch Lautstärkeveränderungen in der Musik ausgelöst wird.

Die sanfte Tiefenbetonung der Musik aktiviert den beruhigenden Parasympathikus: Nach einer meist kurzen Gewöhnung und Anpassungsreaktion kommen Kinder sowie Erwachsene zur Ruhe, werden stiller und zentrierter. Sie kommen bei sich selbst an. Dann wieder stimuliert die Höhenbetonung der Musik den Sympathikus: Die Patienten werden wacher und aufmerksamer; der Hörsinn wird nun zum Fernsinn, mit dem wir die Welt entdecken wollen.

$\mathrm{Zu}$ Beginn der Hörtherapie hören die Patienten über mehrere aufeinanderfolgende Tage täglich Mozart und gregorianische Gesänge mit der beschriebenen Frequenzmodulation der «Klangwippe» und mit einem deutlichen Überwiegen auf Tiefenbetonung und damit Entspannung.

Erfahrungsgemäss bewirkt das Therapiemodul der «Klangwippe» eine Verbesserung der Aufmerksamkeit und Wachheit. Erklärt wird diese empirisch zuverlässig eintretende Wirkung unter anderem mit einer Stimulation des Mittelohrmuskelsystems (und der Schallvorverstärkung im Innenohr). Der Wechsel von Tiefen- und Höhenbetonung regt in diesem Modell die Muskeln hinter dem Trommelfell unwillkürlich zu einem Wechsel von Erschlaffung und Spannung an. Immer wieder werden also «die Ohren gespitzt», verbunden mit einer fokussierten, sprachbezogenen

\section{KARGER}

Fax +497614520714 Information@Karger.d www.karger.com
(๑) 2012 S. Karger GmbH, Freiburg
Dr. med. Dirk Beckedorf

Neukirchstrasse 12, 28215 Bremen, Deutschland

dirk@drbeckedorf.de 
Aufmerksamkeit. Kinder lernen so, selektiv wahrzunehmen und Nutzsignale wie die Stimme des Lehrers in einem Klassenraum von den Störsignalen der Hintergrundgeräusche zu trennen. Sie können sich also allmählich besser konzentrieren und lernen unwillkürlich besser zuzuhören.

\section{Stärkung von Körperspannung und Körperwahrnehmung}

Hinzu kommt ein weiteres zentrales Wirkprinzip der Hörtherapie: Das Zusammenspiel von Knochenleitungshören (Körperschall) und Luftleitungshören wird trainiert und differenziert. Wir hören uns selbst, d.h. unsere eigene Stimme, intensiv über die Übertragung der Kehlkopfschwingungen auf Brustkorb, Halswirbelsäule und Schädel. Die Stimmen anderer Menschen hören wir vor allem via Trommelfell und Mittelohr. Bei der Hörtherapie wird die Musik mit Spezialkopfhörern dargeboten, die neben dem üblichen Weg über das Trommelfell/Mittelohr auch einen Knochenschallgeber enthalten. Über diese zusätzliche Stimulation der Knochenleitung werden die oben bereits beschriebenen körperlichen Aspekte des Hörens intensiviert. Die Patienten spüren sich stärker in sich, in ihrem Körper. Das Körperselbst als Basis des «Ich-Gefühls» wird so gefestigt. Auch funktionale Aspekte der körperlichen Wahrnehmung verbessern sich häufig: Es entwickelt sich eine bessere Spannung im Körperstamm (besserer Tonus); auch Kraftdosierung und motorische Koordination können sich verfeinern.

Im Hörprofil fällt bei vielen Patienten auf, dass ihre Wahrnehmung über Knochen- und Luftleitung mitunter auffallend voneinander abweicht, ohne dass hörorganische Erkrankungen vorliegen. Im Verlauf der auditiven Stimulation gleichen sich in den Hörprofilkontrollen Luftund Knochenleitung aneinander an. Dies fassen wir als eine beginnende Integration von Selbst- und Fremdwahrnehmung auf.
Förderung von Emotion, Motivation und Bindung

Ein weiteres zentrales Prinzip der Hörtherapie ist die allmähliche Betonung des Hörens auf immer höhere Frequenzen. Im Verlauf des ersten Behandlungsabschnitts von 15 Tagen werden häufig nach etwa einer Woche die tiefen Frequenzen aus der Musik von Mozart schrittweise immer stärker herausgefiltert. Oft wird bei den behandelten Kindern durch diese Höhenbetonung eine Anregung der Sprechfreudigkeit und Kontaktfähigkeit bewirkt. Ein typischer Satz der Eltern ist: «Der Mund steht nicht mehr still.» Hinzu kommt nun bei einer Reihe von Kindern auch das Hören einer Aufzeichnung der Stimme der Mutter. Diese liest eine halbe Stunde einen Text vor, den wir aufnehmen. Die Kinder (und auch erwachsene Hörtherapiepatienten) hören anschliessend in mehrfacher Wiederholung diese Aufzeichnung mit einem Hochpassfilter. Die Stimme ist inhaltlich nicht zu verstehen; sie klingt wie ein Gewisper. Wahrnehmbar bleiben Melodie, Klangfarbe und Rhythmus. Offensichtlich regt dieses besondere Hören der Mutterstimme die Kinder an, sich mehr mitzuteilen. Manche werden in dieser Zeit auch etwas anhänglicher, suchen Körperkontakt $\mathrm{zu}$ den Hörtherapieassistentinnen und zur Mutter. Beliebte Spiele sind z.B. «Baby sein», Höhlen bauen usw. In den meisten Fällen intensiviert sich die Bindung zwischen Mutter (Eltern) und Kind. Immer wieder beobachten wir, dass Kinder mit unsicherem Bindungsstil allmählich durch Wiederholung dieser Hörtherapieerfahrung Zeichen einer sicheren Bindung erkennen lassen.

Wir erklären diese vielfältigen Wirkungen mit einer Anregung vorsprachlicher und vorgeburtlicher Hörerfahrungen durch die höhenbetonte Mutterstimme. Der Hörsinn ist in den letzten 3-4 Schwangerschaftsmonaten weitgehend funktionsfähig. Das Kind hört über das
Fruchtwasser und das Knochensystem der Mutter. Wenn es mit seinem Kopf im mütterlichen Becken sicher geborgen liegt, kommt es zu starker Knochenresonanz. Das Baby im Bauch lernt so nicht nur, nach der Geburt die Stimme seiner Mutter zu erkennen, sondern auch die typische Melodie und Klangfarbe der Sprache, die die Mutter spricht. Ein Beleg für diese tiefgehenden Lernerfahrungen bereits im Mutterleib sind z.B. Untersuchungen, die nachweisen, dass neugeborene Babys sprachspezifisch schreien, was sie offensichtlich bereits im Mutterleib gelernt haben.

Das Hören der höhenbetonten Mutterstimme während der Hörtherapie erinnert in unserem Erklärungsmodell an die Bindungs- und Lernerfahrungen des Knochenhörens im Mutterleib.

Viele Erfahrungen weisen darauf hin, dass gerade durch das Hören höhenbetonter Mozart-Musik und der gefilterten Mutterstimme Lernprozesse im zentralen Hörsystem bewirkt werden. Es kommt immer wieder zu einer Reifung der Hörverarbeitung und Hörwahrnehmung im Gehirn, die in den regelmässigen Kontrollen des Hörprofils nachweisbar sind.

Wenn alles gut läuft, dann fangen die Kinder in dem beschriebenen Hörtherapieabschnitt also an, sich und anderen mehr zu vertrauen. Sie sprechen mehr und lernen auch, ihre Impulse besser $\mathrm{zu}$ steuern und $\mathrm{zu}$ kommunizieren. Dies bildet die Basis für einen weiteren Schritt in der Hörtherapie: die aktive Stimm- und Sprecharbeit.

\section{Entwicklung der kommunikativen Fähigkeiten}

Eine vierte Wirkkomponente der Hörtherapie ist die aktive Arbeit, auch audiovokale Übung genannt. Hierbei summen, lesen oder singen die Patienten in ein Mikrofon und hören dabei ihre eigene Stimme in Echtzeit, die aber durch den Klangwandler spezifisch verändert wird. 
Das Summen in der Hörtherapie wird so angeleitet, dass der Klang vor allem über die Schwingung des Knochen-/Skelettsystems entsteht. Der Luftstrom setzt den Kehlkopf in Schwingung und dieser die Wirbelsäule mit dem gesamten Knochensystem.

Da das Summen keinen semantischen Gehalt hat, sondern primär den Eigenklang des Knochen- und Körpersystems erleben lässt, wird mit ihm in besonderer Weise die Selbstwahrnehmung und die Körperresonanz angeregt. Was vorher über den Knochenschallgeber im Kopfhörer stimuliert wurde, wird jetzt durch die eigene Stimme intensiviert. Summen mit dem Knochenklang heisst, mit sich in Kontakt und Resonanz zu sein und darin sich mit sich selbst eins zu erleben. Die positive Körperresonanzerfahrung stärkt das Körperselbst und reichert es mit einem positiven Selbstwertgefühl an. Hinzu kommt das Erleben von Selbstwirksamkeit. Der Klangwandler wird so eingestellt, dass die Klangwippe nur dann umspringt, wenn mit der eigenen Stimme in das Mikrofon gesprochen wird.

Das Nachsprechen von vorgesprochenen Worten und Sätzen sowie das Lesen von Texten ist dann ein nächster Schritt. Hierbei verschiebt und erweitert sich der Aufmerksamkeitsfokus von innen, dem «Ich», mehr nach aussen, auf den nachzusprechenden oder zu lesenden Inhalt und auf die Person, die mir vorspricht und singt. Zuhören und sich selbst zum Ausdruck bringen sind die zu lernenden Inhalte. So kann sich eine das «Ich» und das «Du» integrierende selbstbewusste Kommunikation entwickeln. Ausserdem gibt es noch eine Besonderheit: Jede Sprache hat ihr eigenes Frequenzprofil, in dem sie klingt. A. Tomatis nennt dieses Frequenzprofil das «ethnische Ohr». Durch frequenzspezifische Einstellungen des Klangwandlers kann das Hören der sprachspezifischen Frequenzen einer Sprache gezielt trainiert werden. So entsteht die phonologische Bewusstheit für die Vokale und Konsonanten der jeweiligen Sprache. Sprache kann so leichter und präziser gelernt werden.

\section{Zeitlicher Ablauf}

Ein Basisprogramm der Hörtherapie hat folgenden zeitlichen Aufbau:

- Hörabschnitt: 15 (12 oder 10) Tage täglich $2 \mathrm{~h}$ Hörtherapie mit begleitenden Gesprächen und Hörprofilen.

- Pause von 4-8 Wochen.

- Hörabschnitt: 8 (6,10 oder 12) Tage täglich $2 \mathrm{~h}$ Hörtherapie mit begleitenden Gesprächen und Hörprofilen.

Je nach vorliegendem Problem und Therapiemotiv können sich weitere Hörabschnitte anschliessen.

\section{Indikationen}

Wie die oben dargestellten Interventionsmöglichkeiten und ihre Wirkungen im Therapieprozess vermuten lassen, sind die Behandlungsmöglichkeiten und Anwendungsgebiete vielfältig.

Anwendungsgebiete für die Hörtherapie bei Kindern sind zunächst zentral auditive Wahrnehmungsstörungen, weiterhin sprachliche und motorische Entwicklungsstörungen, Behinderungen (wie z.B. Zustände nach geburtsbedingter
Hirnschädigung oder auch DownSyndrom), Bindungsstörungen, Kontakt- und Verhaltensauffälligkeiten, Aufmerksamkeitsstörungen, Teilleistungsstörungen wie Lese-Rechtschreib-Schwäche oder auch tiefgreifende Entwicklungsstörungen wie Autismus.

Bei den eben genannten möglichen Anwendungsgebieten ist immer im Einzelfall in einem Erstgespräch mit Hörprofil zu klären, in welchem Ausmass eine Störung der Hörverarbeitung und -wahrnehmung sowie der Adaptionsfähigkeit des Mittelohres an den jeweiligen Problemen des Kindes beteiligt sein können.

Die Anwendungsgebiete bei Erwachsenen sind zunächst auch hier Hörprobleme, die z.B. durch Hörsturz, Schwerhörigkeit und Tinnitus entstehen. In vielen Fällen kann hier eine merkliche Verbesserung erreicht werden. Weiterhin gehören die Rehabilitation nach Schlaganfall und Schädelhirntrauma sowie das Spektrum psychosomatischer Symptome wie Schlafstörungen und chronische Schmerzzustände dazu. Emotionale Probleme wie Depression, Burnout und Ängste, ferner Beziehungsschwierigkeiten und Bindungsstörungen können ebenfalls durch die Hörtherapie Hilfe erfahren. Nicht unerwähnt bleiben soll der gesamte sprachliche Bereich wie Verbesserung von Stimme und Gesang und das Erlernen von Fremdsprachen.

\section{Literatur}

Beckedorf D, Müller F: Von der Resonanz zur Bindung. Berlin, Ulrich Leutner, 2010.

Institut Auris Integralis: www.auris-integralis.de. Schweizerischer Berufsverband für Audio-Psycho-Phonologie: $w w w . a-p-p . c h$.

Tomatis AA: Der Klang des Lebens. Hamburg, Rowohlt, 1995. 